\title{
Tratamento Trombolítico no Acidente Vascular Cerebral Isquêmico
}

\author{
Ricardo Afonso Teixeira', Leonardo de Deus Silva², Valério Ferreira ${ }^{3}$
}

\section{RESUMO}

O acidente vascular cerebral isquêmico (AVCi) é uma das mais importantes causas de mortalidade e morbidade em todo o mundo. A atual disponibilidade de métodos mecânicos e farmacológicos para o restabelecimento do fluxo sangüíneo cerebral em uma artéria obstruída tem possibilitado a mudança da historia natural do AVCi. Neste artigo discutimos as principais estratégias de recanalização arterial no AVCi agudo. A trombólise endovenosa com rt-PA é atualmente a terapêutica mais estudada e com maior evidência de benefício em promover recanalização arterial. Entretanto, uma importante limitação da trombólise endovenosa é a necessidade de seu uso dentro de uma janela temporal de 3 horas após o início dos sintomas, um dos motivos pelos quais outras estratégias foram desenvolvidas. Trombólise intra-arterial isolada ou combinada à endovenosa, métodos endovasculares como angioplastia e colocação de stent, e novas drogas trombolíticas são outros métodos discutidos. Alguns destes já fazem parte da rotina de muitos centros, enquanto outros ainda precisam ser testados em estudos mais robustos.

Descritores: Acidente vascular cerebral, Trombólise, rt-PA

\section{SUMMARY}

Acute ischemic stroke (AIS) is one of the most important cause of mortality and morbidity around the world. The recent availability of mechanical and pharmacological methods to achieve reestablishment of cerebral blood flow in an occluded vessel has modified the natural course of AIS. In this article we discuss the main strategies of arterial recanalization in AIS. Intravenous thrombolysis with rt-PA is currently the most studied treatment, with proved efficacy but presenting some limitations. New strategies were developed in order to overcome these limitations, especially the short 3-hour temporal window. Isolated or combined intraarterial thrombolysis, endovascular treatment such as angioplasty and stent placement, and new thrombolytic drugs are also discussed. Some of these methods are already routinely used in many centers, and others still have to be tested in large studies.

Key words: Acute ischemic stroke, Thrombolysis, rt-PA.

\section{1 - INTRODUÇÃO}

No mundo ocidental, as doenças cerebrovasculares são responsáveis pelo comprometimento de uma boa parte da população economicamente ativa. Estima-se que nos países industrializados, 300 a 500 pessoas para cada 100 mil habitantes são vítimas desse grupo de doenças ${ }^{1}$. Isto concorre para o fato de que as doenças cerebrovasculares, especialmente o acidente vascular cerebral (AVC), seja a segunda maior causa de morte e a primeira causa de incapacidade física permanente no mundo ${ }^{2}$. Os AVCs de etiologia isquêmica (AVCi) representam 70 a $80 \%$ dos casos, e portanto, são os principais alvos para o desenvolvimento de terapêuticas que visem a redução da mortalidade e incapacidade física decorrentes das doenças cerebrovasculares.
Não está no escopo deste artigo revisar os mecanismos básicos e nem os fatores de risco associados ao AVCi, uma vez que é fácil encontrar na literatura médica revisões sólidas sobre tais assuntos. Nosso intuito é discutir o tratamento agudo desta patologia, com ênfase no que é hoje considerado o maior modificador da história natural do AVCi: o tratamento trombolítico.

\section{2 - O QUE É TROMBÓLISE?}

Trombólise, como o próprio nome já define, diz respeito à lise de um trombo. Como o AVC isquêmico decorre da obstrução de um vaso arterial cerebral, seja por um êmbolo proveniente de um sítio proximal, seja por um trombo formado in situ, a idéia fundamental desta

\footnotetext{
1 - Neurologista, Coordenador do Programa AVC, Hospital Santa Luzia, Brasília-DF, Professor Doutor da Universidade Católica de Brasília.

2 - Neurologista, Pós-Graduando do Departamento de Neurologia, UNICAMP, Campinas-SP
}

3 - Neuroradiologista Intervencionista, Hospital Santa Luzia, Brasília-DF 
terapia é a desobstrução desta artéria antes que haja um grau de lesão tecidual irreversível ${ }^{3,4}$.

Considerando-se que o fluxo sangüíneo cerebral (FSC) normal é no mínimo de $55 \mathrm{ml} / 100 \mathrm{~g} / \mathrm{min}$, o tecido neuronal começa a apresentar variados graus de disfunção quando esse valor se reduz. Vários estudos animais, corroborados atualmente por estudos de neuroimagem funcional, sugerem que a síntese protéica celular normal começa a diminuir com valores de FSC próximos ao normal (40 a $50 \mathrm{ml} / 100 \mathrm{~g} / \mathrm{min}$ ), seguindo de glicólise anaeróbica (35ml/ $100 \mathrm{~g} / \mathrm{min})$, perda de transmissão sináptica $(20 \mathrm{ml} / 100 \mathrm{~g} /$ min) e, finalmente, despolarização anóxica das membranas celulares com cessação de atividade elétrica (15 a $17 \mathrm{ml} / 100 \mathrm{~g} / \mathrm{min}$ ). Valores de FSC regional abaixo de $12 \mathrm{ml} / 100 \mathrm{~g} / \mathrm{min}$ resultam em necrose e morte celular, enquanto déficits transitórios ocorrem quando o FSC se mantém acima de $22 \mathrm{ml} / 100 \mathrm{~g} / \mathrm{min}^{5}$. Os valores de FSC entre 12 e 22ml/100g/min são de extrema importância na trombólise, já que o tecido neuronal irrigado através do FSC nesse intervalo constitui a preciosa penumbra isquêmica. A penumbra isquêmica representa uma área de tecido neuronal comprometido, disfuncional, mas com grande capacidade de recuperação, desde que o FSC seja restaurado rapidamente. Atualmente, a terapêutica mais eficaz na restauração do FSC normal é a trombólise.

\section{3 - HISTÓRICO}

Visto que o mecanismo patogênico do AVCi é a obstrução tromboembólica de uma artéria cerebral, o uso de um agente com propriedades de lise desse trombo seria a escolha lógica. Foi ainda na década de 50 quando ocorreram os primeiros casos de AVCi em que um agente trombolítico foi usado, mas sem sucesso. A ausência de tomografia computadorizada (TC) foi um das causas desse insucesso, pois alguns pacientes com hemorragia intracraniana foram tratados com trombólise, piorando sobremaneira a evolução natural desses casos. Em 1985, Zivin et al. demonstraram que o ativador tissular de plasminogênio recombinante (rt-PA) foi eficaz em promover recanalização arterial em modelos experimentais de ratos ${ }^{6}$. Desde então, a comunidade médica foi tomada por um entusiasmo coletivo no intuito de se estabelecer um tratamento eficaz e seguro, uma droga que promovesse a recanalização arterial com mínimos efeitos colaterais.

Tentando extrapolar os resultados obtidos no tratamento do infarto agudo do miocárdio (IAM), vários estudos com estreptoquinase foram planejados e executados. Entretanto tais estudos evidenciaram altos níveis de hemorragia intracraniana e resultados desfavoráveis. Os estudos Multicenter Acute Stroke TrialEurope (MAST-E), o Australia Streptokinase (ASK), e o Multicenter Acute Stroke Trial-Italy (MAST-I), avaliaram o uso de estreptoquinase endovenosa na dose de $1.5 \mathrm{M} \mathrm{UI}$ em pacientes com AVCi na fase hiperaguda, e tiveram que ser prematuramente encerrados em razão da excessiva mortalidade e das hemorragias intracranianas sintomáticas nos pacientes tratados, quando comparados com o grupo que fez uso de placebo ${ }^{7-9}$.

A estreptoquinase foi a primeira droga trombolítica utilizada em seres humanos, mas sua eficácia no AVCi não pôde ser comprovada. Vários fatores contribuíram para o insucesso da estreptoquinase. Os pacientes foram tratados em média 4,2 horas após a instalação dos sintomas, janela temporal maior que a usada nos estudos posteriores com rt-PA. Os critérios de inclusão também não foram tão rigorosos quanto aos estudos com rt-PA, e pacientes com sinais precoces de infarto maior à TC não foram excluídos. Um outro aspecto negativo na metodologia desses estudos foi que a dose de estreptoquinase utilizada foi a mesma usada nos estudos de IAM, e não houve escalonamento prévio da dose a fim de se estabelecer qual seria a menor dose eficaz. Tais críticas suscitam a possibilidade de uma eventual eficácia da estreptoquinase no AVCi, caso a mesma seja utilizada em doses menores e numa janela de tempo mais curta. Esta hipótese ainda não foi testada.

Baseados no sucesso do rt-PA em promover recanalização em modelos experimentais, estudos pilotos com esta droga foram iniciados na década de 80 a fim de se estabelecer o tempo ideal e a dose segura no AVCi ${ }^{10-}$ 12. Os resultados dos estudos pilotos foram fundamentais para o planejamento do NINDS $t$-PA Stroke Study ${ }^{13}$, o primeiro grande estudo randomizado que demonstrou benefício do uso de um agente trombolítico no AVCi na fase hiperaguda. Foram basicamente os resultados desse estudo que fundamentaram a aprovação do uso do rt-PA pelo Food and Drug Administration (FDA) para o tratamento do AVCi, desde que usado numa janela de até 3 horas do início da instalação do quadro.

\section{4-TROMBÓLISE ENDOVENOSA}

O restabelecimento do FSC em uma artéria obstruída é fundamental para o salvamento do tecido neuronal em risco. Logo após a obstrução do vaso, mecanismos fibrinolíticos endógenos entram em ação, culminando com a ativação do plasminogênio em plasmina que, de uma forma geral, quebra a malha de fibrina, dissolvendo o trombo. Os ativadores fisiológicos do plasminogênio são encontrados no soro em quantidades muito baixas, em concentração 100.000 vezes menor que a concentração do plasminogênio, e incluem o ativador do plasminogênio tissular e o ativador uroquinase do plasminogênio. Entretanto, esse mecanismo fibrinolítico endógeno não é tão eficaz. Recanalização espontânea ocorre em uma minoria de pacientes com AVCi ${ }^{14}$, e as drogas trombolíticas passam a ser grande aliados para a restauração do fluxo sangüíneo.

O estudo NINDS rt-PA Stroke Study foi executado em duas partes ${ }^{13}$, e foi o primeiro estudo randomizado a demonstrar o benefício do uso do rt-PA em até 3 horas da instalação do quadro. A primeira parte do estudo foi desenhada com o objetivo de se evidenciar a proporção de pacientes com melhora ou resolução completa e precoce dos sintomas neurológicos, o que era definido como uma redução de 4 pontos ou mais na escala NIHSS (escala de déficit neurológico), dentro de um período de 24 horas. Pacientes foram randomizados a receber placebo ou rt-PA na dose de $0,9 \mathrm{mg} / \mathrm{Kg}$, sendo $10 \%$ da dose administrada em bolus e o restante através de infusão contínua em 1 hora sem exceder a dose máxima de $90 \mathrm{mg}$. Tais valores foram determinados a partir dos estudos pilotos que precederam o estudo NINDS ${ }^{10-11}$. Pacientes com todos os tipos de AVC isquêmico foram 
incluídos, desde que a duração dos sintomas não ultrapassasse 180 minutos. Foram incluídos 291 pacientes, 139 (48\%) com até 90 minutos de início dos sintomas. Dos 144 pacientes randomizados para o tratamento com rt-PA, 67 (46\%) apresentaram melhora precoce, enquanto 57 (39\%) dos 147 que receberam placebo apresentaram o mesmo grau de melhora (risco relativo 1.2; $p=0.21$ ). Hemorragia intracraniana sintomática aconteceu em $6 \%$ dos pacientes que receberam rt-PA e não foi observada em nenhum paciente randomizado para o placebo.

Os resultados da primeira parte do estudo não foram suficientes para se estabelecer um beneficio claro, tendo sido necessária a extensão do estudo, utilizando a mesma droga na mesma dose, mas com objetivos um pouco diferentes. Desta vez, a variável testada foi uma boa recuperação funcional dos pacientes três meses após o tratamento, definida como: escala NIHSS £ 1, Índice de Barthel ${ }^{3} 95$ (mensura a independência para atividades de vida diária), Escala de Rankin Modificada £ 1 (mensura a incapacidade física), e escore de 1 na Escala de Evolução de Glasgow (mensura status funcional).

Nesta segunda parte do estudo, 333 pacientes foram randomizados para receber rt-PA ou placebo, e 163 (49\%) pacientes foram tratados com até 90 minutos da instalação do quadro. Os pacientes que foram randomizados para receber rt-PA apresentaram resultados melhores que os pacientes submetidos ao tratamento com placebo. O odds ratio para um resultado favorável no grupo tratado com rt-PA foi 1,7 (Intervalo de confiança [IC]: 1.2-2.6; $p=0.008$ ). Ao se analisar a diferença absoluta das proporções de bons resultados nos dois grupos, seriam necessários oito pacientes tratados com rt-PA para que um paciente adicional apresentasse resolução completa ou recuperação com déficits mínimos. Numa análise combinada das partes 1 e 2 do estudo NINDS, os benefícios do rt-PA em 3 meses foram comprovados em cada escala de avaliação funcional utilizada.

Hemorragia intracraniana sintomática em até 36 horas após o início do quadro foi observada em $7 \%$ dos pacientes do grupo tratado com rt-PA comparado a apenas $1 \%$ dos pacientes que fizeram uso do placebo na parte 2 do estudo. A análise combinada das duas partes do estudo evidenciou uma incidência de hemorragia intracraniana sintomática em $6,4 \%$ dos pacientes que fizeram uso do rt-PA comparado com $0,6 \%$ dos pacientes tratados com placebo $(p<0.001)$. Em uma análise multivariada, os únicos fatores que contribuíram independentemente para um risco maior de sangramento foram a gravidade do déficit clínico medido pela NIHSS (>20) $(O R=1.8 ; I C: 1.2-2.9)$, e a presença de edema cerebral evidenciado por hipodensidade hiperaguda ou efeito de massa (OR=7.8; IC:2.2-27.1). Entretanto, os benefícios do tratamento se mantiveram nesse grupo de pacientes que apresentavam esses achados clínicos ou radiológicos de "maior risco de sangramento", com uma melhor recuperação funcional em 3 meses e semelhante probabilidade de óbito ou incapacidade física grave, quando comparado ao grupo placebo.

Em uma análise diferenciada de subtipos de AVCi, os benefícios do uso do rt-PA no estudo NINDS foram observados em pacientes representativos de diferentes graus de comprometimento neurológico assim como em todos os tipos de AVCi: aterotrombose de grandes vasos, doença de pequenos vasos e AVCi cardioembólico. A probabilidade do uso do rt-PA resultar em recuperação completa ou quase completa no grupo tratado em até 90 minutos da instalação do quadro (OR=2.11; IC:1.33-3.35) foi maior que a do grupo tratado entre 90 e 180 minutos (OR=1.69; IC:1.09-2.62), quando comparados ao grupo placebo. Os benefícios do tratamento com rt-PA mantiveram-se por no mínimo 1 ano.

Outros estudos com rt-PA foram realizados no intuito de se estudar a possibilidade do uso dessa droga em uma janela de tempo superior a 3 horas. Dois grandes estudos randomizados foram realizados na Europa com esse objetivo. O primeiro estudo, European Cooperative Acute Stroke Study (ECASS) foi publicado dois meses antes da publicação do estudo NINDS e sete meses antes da aprovação do uso do rt-Pa pelo FDA ${ }^{15}$. O ECASS foi o primeiro grande estudo multicêntrico, duplo-cego, randomizado, que comparou o uso de rt-PA com placebo.

O ECASS incluiu pacientes maiores de 18 anos, com diagnóstico clínico de AVCi moderado a grave e com até 6 horas de instalação do quadro clínico. Um total de 620 pacientes foram randomizados para receber rt-PA ou placebo. A dose recomendada para ser administrada no grupo que receberia rt-PA foi de $1,1 \mathrm{mg} / \mathrm{kg}$. Foram excluídos pacientes que apresentassem coma, hemiplegia associada a desvio conjugado do olhar, afasia global, AVC do sistema vétebro-basilar ou TC inicial evidenciando hipodensidade em mais de um terço do território da artéria cerebral média. Os objetivos primários foram a análise da melhora funcional definida por: Índice de Barthel em 15 pontos e Escala de Rankin Modificada em 1 ponto, com a avaliação tendo sido realizada 90 dias depois do tratamento. O objetivo secundário foi a análise da mortalidade em 30 dias.

Os resultados do ECASS não foram empolgantes, pois não houve diferença significativa na análise dos resultados dos objetivos primários entre o grupo rt-PA e placebo. A mortalidade em 90 dias foi de $22,4 \%$ no grupo tratado com rt-PA e $16 \%$ no grupo placebo $(p=0.04)$. Entretanto, houve inúmeras violações de protocolo nesse estudo. Dos pacientes incluídos, 18\% apresentavam algum critério de exclusão. A violação de protocolo mais comum foi a inclusão de pacientes com hipodensidade maior que $30 \%$ da área da artéria cerebral média. Em uma análise diferenciada, excluindo os pacientes com violação do protocolo, observou-se uma diferença significativa nos resultados da Escala Rankin Modificada, favorecendo o grupo que recebeu rt-PA. A mortalidade não foi diferente entre os dois grupos. Infartos hemorrágicos aconteceram com maior freqüência no grupo placebo $(30,3 \% \times 23 \%, p<0.001)$. Entretanto, hematomas intracranianos foram mais comuns no grupo que recebeu rt-PA $(19.8 \% \times 6.5 \%, p<0,005)$.

Com o objetivo de excluir o excesso de violações de protocolo e responder outras questões, foi planejado o ECASS $/ I^{16}$. O estudo foi também duplo-cego, randomizado, multicêntrico, e testou a eficácia e segurança do rt-PA em até 6 horas da instalação do quadro clínico. Diferente do ECASS I, a dose de rt-PA utilizada foi de $0,9 \mathrm{mg} / \mathrm{kg}$ e antes do início do estudo os centros envolvidos foram instruídos 
a cumprir rigorosamente os critérios de inclusão e exclusão, tendo sido inclusive treinados para uma avaliação mais precisa da TC de crânio. Mesmo com todos esses cuidados, houve 72 violações de protocolo no universo de 800 pacientes randomizados.

O objetivo principal desse estudo era avaliar a Escala de Rankin Modificada ( $m R S$ ) em 90 dias, sendo os resultados dicotomizados em favoráveis (escores $0-1$ ) ou desfavoráveis (escores 2-6). Nesta análise, não houve diferença significativa na proporção de resultados favoráveis ou desfavoráveis entre os dois grupos (40,3\% x 36,6\%). Entretanto, numa análise post-hoc, foram redefinidos 2 grupos: fisicamente independentes ( $m R S$ $0-2$ ) e dependentes ( $m R S$ 3-6). Esta análise revelou um maior número de pacientes independentes entre aqueles que foram tratados com rt-PA, quando comparado com o grupo que recebeu placebo $(54.3 \% \times 46 \% ; p=0.024)$. A incidência de hemorragia intracraniana sintomática foi significativamente maior no grupo tratado com rt-PA $(8,8 \%$ $x 3,4 \%$ ), mas não houve diferença significativa na mortalidade em 30 ou 90 dias entre os dois grupos.

Outro estudo que avaliou a eficácia do rt-PA foi o "Alteplase Thrombolysis for Acute Noninterventional Therapy in Ischemic Stroke" (ATLANTIS) ${ }^{17}$. Tal estudo foi duplo-cego, randomizado, e inicialmente tentou estudar o uso do rt-PA em pacientes com AVCi até 6 horas de instalação do quadro. Após randomização de 142 pacientes, o comitê de segurança do estudo mudou os critérios de inclusão para até 5 horas, pois havia uma grande preocupação com o grupo de pacientes com 5-6 horas de instalação do quadro. Após a aprovação do uso do rt-PA em 1996 dentro da janela de 3 horas, o protocolo desse estudo foi mais umas vez alterado e focalizou a análise de um eventual benefício em pacientes tratados com 3-5 horas do ictus. Em 1998 esse estudo foi prematuramente interrompido baseado no parecer do comitê de segurança que concluiu a improbabilidade do estudo em demonstrar benefício com o tratamento trombolítico.

\section{TROMBÓLISE INTRA-ARTERIAL}

Paralelamente ao intenso esforço dispendido na organização e execução dos estudos em trombólise endovenosa, houve também um grande progresso na tecnologia de dispositivos endovasculares para realização de trombólise intra-arterial.

A partir da década de 80 , inúmeras séries de pacientes com AVCi tratados com trombólise intra-arterial e dois estudos randomizados foram publicados ${ }^{18}$. As drogas mais amplamente usadas foram a uroquinase, prouroquinase e rt-PA. Não há evidências sobre a superioridade de uma medicação sobre a outra, já que nenhum estudo foi desenhado para responder esta questão. Os estudos referentes a AVCi da circulação anterior demonstraram taxas de recanalização (parcial ou completa) geralmente acima de $70 \%$, hemorragias sintomáticas de $0-17 \%$ e bom prognóstico acima de $50 \%$.

Pacientes com AVCi por oclusão do sistema vértebrobasilar representam um cenário bem distinto, pois geralmente apresentam péssimo prognóstico com taxas de mortalidade em torno de $70-80 \% \%{ }^{18-20}$. Quando a recanalização é alcançada, a taxa de sobrevida é de 55-
$75 \%$, em oposição a $0-10 \%$ de sobrevida no caso de uma obstrução basilar não recanalizada.

Várias séries de pacientes revelaram a trombólise intra-arterial como uma valiosa arma nos casos de AVCi de circulação posterior, com taxas de recanalização de $70 \%$ em média ${ }^{18,19}$. Nestes estudos, o tratamento trombolítico foi administrado tardiamente (média de 6 horas após início dos sintomas) quando comparado a pacientes com AVCi de circulação anterior. A janela temporal para a trombólise no AVCi de circulação posterior ainda não foi definida mas pode chegar a 12 horas.

Nos dois estudos randomizados que avaliaram a eficácia da trombólise intra-arterial, a droga usada foi a prouroquinase, foram incluídos apenas pacientes com oclusão da artéria cerebral média com menos de 6 horas do início do quadro, e não foi permitida a manipulação mecânica do trombo. No PROACT // (o maior deles), 121 pacientes receberam tratamento trombolítico associado a heparina em baixas doses e 59 receberam apenas heparina ${ }^{21}$. Recanalização completa ou parcial foi obtida em $66 \%$ dos pacientes no grupo que recebeu 0 trombolítico, comparado a $18 \%$ no grupo que recebeu apenas heparina $(p<0.001)$. Após três meses do tratamento, $40 \%$ dos pacientes tratados com trombolítico apresentavam independência funcional comparado a $25 \%$ no grupo placebo $(\mathrm{p}=0.04)$. O PROACT I também demonstrou maior taxa de recanalização entre os pacientes que receberam trombolítico (58\% no grupo tratado e $14 \%$ no grupo placebo) ${ }^{14}$.

A trombólise intra-arterial já é um tratamento justificável do ponto de vista de eficácia e segurança, e é considerado por grande parte dos especialistas como método de eleição para pacientes com AVCi da circulação anterior com duração dos sintomas entre 3-6 horas, naqueles com evidência de oclusão de ramos principais da artéria cerebral média mesmo ainda dentro da janela de 3 horas, e nos pacientes com AVCi por oclusão do sistema vértebro-basilar, onde as evidências de eficácia são muito maiores do que com a trombólise endovenosa ${ }^{22,23}$.

\section{Vantagens da trombólise intra-arterial}

1. Diagnóstico mais seguro devido à identificação de oclusão arterial pela arteriografia. Evita a administração de trombolítico a pacientes com falsos AVCs (e.g.; crises conversivas) e com lesões que sabidamente não respondem ao trombolítico (e.g.; extensas estenoses sem trombose).

2. Maior eficácia na recanalização de oclusão de grandes artérias. A infusão seletiva do trombolítico permite uma concentração alta da droga no trombo.

3. Risco de hemorragia intracraniana menor ou pelo menos igual à trombólise endovenosa. Devido à infusão seletiva do trombolítico, a dose usada é menor e com menos efeitos sistêmicos.

4. Possibilidade de manipulação mecânica do trombo ou até mesmo realização de angioplastia (ver item 6.3).

\section{Desvantagens da trombólise intra-arterial}

1. Potencial demora para o início do tratamento devido à complexa logística de uma angiografia.

2. Riscos e custos adicionais de um procedimento invasivo. 
3. Disponibilidade de poucos serviços hospitalares estruturados com neuroradiologia intervencionista 24 horas.

\section{META-ANÁLISES}

Duas grandes meta-análises foram realizadas para avaliar o benefício do tratamento trombolítico no AVCi. A primeira delas publicada em 1999 incluiu 2044 pacientes dos estudos NINDS e ECASS / e // (1034 pacientes submetidos a trombólise) ${ }^{24}$. Os pacientes tratados com trombolítico apresentaram uma leve tendência de redução da mortalidade quando tratados até 3 horas do início dos sintomas (OR=0.91; IC: 0.63-1.32). Já a variável morte ou dependência foi reduzida em $37 \%$ entre aqueles tratados com trombolítico, independente da janela temporal $(\mathrm{OR}=0.63$; IC: 0.53-0.76). Hemorragia intracraniana foi mais comum entre os pacientes que receberam o tratamento (144/1034) do que entre aqueles que receberam placebo (43/1010).

A outra meta-análise é do banco de dados Cochrane e avaliou 17 estudos incluindo aqueles que usaram estreptoquinase além dos estudos intra-arteriais randomizados (PROACT I e II) ${ }^{25}$. Apesar dos pacientes submetidos a trombólise demonstrarem um aumento no número de hemorragias intracranianas sintomáticas $(\mathrm{OR}=3.53$; IC: $2.79-4.45)$ e fatais $(\mathrm{OR}=4.15 ; \mathrm{IC}: 2.96-5.84)$, menos pacientes apresentaram morte ou dependência ao final de 3-6 meses (OR=0.83, IC: 0.73-0.94). Quando o tratamento foi realizado nas primeiras 3 horas após o início dos sintomas, a redução de morte ou dependência foi ainda maior (OR=0.58, IC: 0.46-0.74). Avaliando-se somente os pacientes tratados com rt-PA, evita-se morte ou dependência em 57/1000 pacientes tratados dentro das 6 horas do início dos sintomas, e em 140/1000 se antes das três horas, um resultado de alto impacto clínico.

\section{6 - NOVAS PERSPECTIVAS}

\section{1 - OUTROS AGENTES TROMBOLÍTICOS}

Estreptoquinase e uroquinase são os agentes fibrinolíticos de primeira geração e apesar de serem efetivos, não têm especificidade para fibrina, criando um estado trombolítico sistêmico ${ }^{26}$.

Os agentes trombolíticos de segunda geração (alteplase e prouroquinase) já são seletivos à fibrina, e apesar de terem sido desenvolvidos para reduzir os efeitos sistêmicos, as doses necessárias para recanalização com estas drogas podem levar a uma redução do plasminogênio e fibrina sistêmicos ${ }^{26}$.

Outros agentes com capacidade de recanalização foram, ou estão sendo testados no sentido de se encontrar a maior potência trombolítica possível sem aumentar o risco de sangramento.

\section{Ancrod}

É uma droga derivada do veneno de uma cobra existente na Malásia. Seu comportamento biológico é o de uma serase com propriedades de retirar o fibrinogênio do sangue. O STAT é um grande estudo duplo-cego, randomizado e multicêntrico, que avaliou a eficácia do ancrod no AVCi hiperagudo ${ }^{27}$. Nesse estudo, foram randomizados 500 pacientes para receber ancrod ou placebo, sendo o início do tratamento com até 3 horas da instalação do quadro. Os pacientes que receberam ancrod apresentaram menor incapacidade física após 3 meses do tratamento, comparados ao grupo que recebeu placebo. Houve também maior tendência a sangramento intracraniano no grupo que recebeu a droga do que no grupo placebo. Em uma recente revisão do banco de dados Cochrane, concluiu-se que apesar do ancrod ser promissor, ainda não existem dados suficientes para garantir o seu uso em AVCi na fase hiperaguda ${ }^{28}$.

\section{Estafiloquinase}

Trata-se de uma quinase com altíssima seletividade para fibrina. É uma droga promissora, pois seu mecanismo de ação é voltado para o plasminogênio ligado a fibrina parcialmente degradada e a plasmina, facilitando sua ação sobre o trombo em lise. Até o momento, esta droga só foi utilizada em estudos experimentais ${ }^{29}$.

\section{Tenecteplase}

É uma droga ativadora do plasminogênio, 14 vezes mais seletiva para a fibrina que o alteplase e também apresenta uma relativa resistência ao inibidor do ativador de plasminogênio ( $P A /)$. Apresenta também uma meia vida maior, sendo possível seu uso em bolus. Esta droga já foi utilizada em alguns estudos de IAM e parece não estar associada com uma maior chance de hemorragia intracraniana. Para o tratamento do AVCi, esta droga ainda não foi testada em humanos, mas estudos com ratos já demonstram alguns benefícios ${ }^{30}$. Um estudo experimental testou a associação de tenecteplase-alteplase, com bons resultados ${ }^{31}$.

\section{Desmoteplase}

Desmoteplase é um outro ativador do plasminogênio gerado por biotecnologia a partir de células de ovários de hamsters chineses. O composto original foi isolado na saliva de um morcego chamado Desmodus rotundus. É provável que esta droga tenha uma afinidade seletiva pela fibrina maior que o alteplase, e, portanto, com menor probabilidade de transformação hemorrágica do AVCi. Já foi realizado um estudo de fase IIA com esta droga em IAM, e um estudo em AVC, denominado "Dose Escalation Study of Desmoteplase in Acute Ischemic Stroke" (DEDAS), está sendo planejado nos Estados Unidos ${ }^{29}$.

\section{Reteplase}

Esforços da bioengenharia para criação de mutantes moleculares do t-PA mais eficazes levaram ao desenvolvimento do reteplase obtido através da Escherichia coli. Tem uma menor afinidade de ligação às células endoteliais e monócitos, e uma maior meia-vida comparado ao alteplase ${ }^{32}$. Estudos realizados com pacientes com infarto agudo do miocárdio revelaram maior potência trombolítica que o alteplase ${ }^{33}$. Também apresenta uma menor especificdade pela fibrina e nenhum grande estudo em AVC usou a droga como trombolítico de escolha.

\section{Abciximab}

Abciximab é um bloqueador dos receptores Ilb/IIla (GPIlb-Illa), que são os mediadores finais da agregação 
plaquetária intermediada pelo fibrinogênio. É um fármaco amplamente usado na cardiologia para redução da incidência de complicações isquêmicas periprocedurais em angioplastia/stent coronarianos e, apesar de ser considerado um agente antitrombótico, há um corpo crescente de evidências demonstrando seu efeito trombolítico ${ }^{34,35}$.

O único estudo randomizado que avaliou o abciximab no AVCi demonstrou ser uma droga segura mas com eficácia muito discreta. Nenhum dos pacientes que recebeu a droga apresentou hemorragia intracraniana sintomática e ainda houve uma tendência a um melhor prognóstico entre estes pacientes, comparado ao grupo que recebeu placebo ${ }^{36}$.

Várias pequenas séries de pacientes têm mostrado um impressionante potencial do abciximab em estabelecer a recanalização imediatamente após o insucesso de terapia trombolítica (trombólise de salvamento) ${ }^{37-39}$. A necessidade de novos estudos controlados do abciximab no AVCi é eminente. No futuro pode vir a ser uma droga que possa ser usada numa janela temporal mais prolongada e eventualmente em combinação com outras drogas trombolíticas.

\section{2 - TERAPIAS COMBINADAS}

Trombólise Endovenosa + Intraarterial

A combinação da trombólise endovenosa e intraarterial parte do princípio do potencial aproveitamento do que há de melhor em cada uma destas modalidades: a rapidez de início do tratamento endovenoso associado à melhor definição diagnóstica e efetividade do tratamento intra-arterial. Após exclusão inicial de hemorragia intracraniana, inicia-se a trombólise endovenosa enquanto aprimora-se o diagnóstico (angiografia precedida ou não por outros exames de neuroimagem), e na presença de oclusão arterial que sabidamente responde melhor à trombólise arterial (e.g.; oclusão de tronco da artéria cerebral média), interrompe-se a trombólise endovenosa e inicia-se a intra-arterial. Alguns estudos já mostraram resultados bastante encorajadores com esta estratégia, mas estudos controlados com maior número de pacientes precisam ser realizados para melhor apreciação ${ }^{40-43}$.

\section{Trombólise Endovenosa + Doppler Transcraniano}

Já existe um grande corpo de evidências sobre o efeito trombolítico do ultrasom, sobretudo em baixas freqüências (<300 kHz). O mecanismo de ação mais provável é a facilitação da quebra de ligações moleculares dos polímeros de fibrina, aumentando assim a superfície de exposição do trombo aos trombolíticos endógenos ou exógenos ${ }^{44-46}$.

Algumas séries de pacientes com AVCi hiperagudo submetidos a insonação prolongada da artéria obstruída sugerem que o doppler trancraniano (DTC) exerce efeito potencializador na recanalização arterial, com ou sem o uso concomitante de trombolíticos ${ }^{47-51}$. Esta é uma estratégia terapêutica bastante interessante devido ao baixo custo e fácil disponibilização do DTC. O impacto desta terapia combinada será melhor demonstrada em estudos randomizados que já estão em andamento.

\subsection{MÉTODOS MECÂNICOS}

A manipulação mecânica do trombo apresenta duas potenciais vantagens em relação à trombólise química: maior rapidez e menor chance de sangramento secundária ao procedimento.

A simples manipulação do trombo com um microcateter pode promover recanalização do vaso ocluído. A técnica aparentemente é mais eficaz quando associada à trombólise química, já que a fragmentação do trombo permite uma maior superfície de contato do trombo com a droga trombolítica. Nestes casos o trombolítico pode ser usado em doses menores e é de grande utilidade devido à potencial embolização distal pós-manipulação do trombo ${ }^{52}$.

Vários dispositivos têm sido desenvolvidos com o objetivo de quebrar ou até mesmo retirar o trombo com a maior rapidez e menores riscos de complicações possíveis. Entre estes dispositivos destacam-se microbalões e microcateteres com alças de sucção ou captura de trombos, além de dispositivos para ablação do trombo através de ultrasom ou laser. Resultados preliminares sugerem que esta é uma modalidade de tratamento que só tende a crescer nos próximos anos ${ }^{53,54}$.

\section{Angioplastia Transluminal Percutânea}

Uma alternativa recente para se obter recanalização arterial em pacientes com AVCi é o tratamento endovascular com angioplastia com ou sem stent. $\mathrm{O}$ benefício do uso de trombolítico por via intra-arterial através de cateterização percutânea já foi comprovado em pacientes selecionados, entretanto não é isento de complicações hemorrágicas e nem garante um resultado sempre favorável ${ }^{55,56}$. Uma das razões mais importantes para a eventual ineficácia do tratamento trombolítico é a resistência à trombólise de um êmbolo de maiores dimensões ou de uma oclusão aterotrombótica, não permitindo uma recanalização adequada e, quando permite, apresenta grandes chances de reoclusão ${ }^{57}$.

Em um estudo recente, Nakano e colaboradores evidenciaram benefício no uso da angioplastia transluminal percutânea (ATP) como tratamento isolado ou associado à trombólise local com dose menor de trombolítico, em pacientes com oclusão aguda do tronco da artéria cerebral média ${ }^{58}$. Nesse estudo foi observado um nível de hemorragia intracraniana no grupo tratado com ATP semelhante ao do grupo não tratado do estudo $P R O A C T$. Esses achados sugerem que a recanalização mecânica através da ATP pode ser uma ótima alternativa para a remoção do trombo, sem aumentar a incidência de complicações hemorrágicas.

Gupta e colaboradores demonstraram recentemente que a angioplastia de urgência é tecnicamente possível, mas observaram um nível inaceitável de complicações. A seleção dos pacientes, o tempo do procedimento e as complicações médicas peri-procedimento foram os fatores críticos que mais contribuíram para a mortalidade e morbidade encontradas ${ }^{59}$. Um outro estudo, com pequeno número de pacientes, sugere que a angioplastia de urgência pode ser uma alternativa após ineficácia da trombólise química ${ }^{60}$.

Portanto, trata-se de uma estratégia de tratamento próspera, seja isoladamente através da restauração 
mecânica do fluxo sanguíneo, seja associada ao uso de drogas trombolíticas. São necessários o planejamento e execução de estudos maiores com critérios de inclusão bem definidos, a fim de se avaliar o real benefício desse método em pacientes com AVCi hiperagudo.

\section{NEUROIMAGEM E SELEÇÃO DE PACIENTES PARA TROMBÓLISE}

O manejo clínico do paciente com suspeita de AVCi na fase hiperaguda requer inicialmente a pronta confirmação do evento isquêmico, além da definição do tipo de AVC (lacunar, embólico, fronteira arterial), do território arterial acometido (grandes vasos, pequenos vasos), e se possível também o mecanismo etiológico envolvido (tromboembólico, cardioembólico, lacunar, hipotensão).

Devido à grande heterogeneidade dos pacientes com AVCi, é absolutamente necessária a definição de critérios cada vez mais refinados para seleção dos pacientes em que o tratamento trombolítico é eficaz. Evitaria-se assim o aumento do risco de sangramento intra- e extracraniano em pacientes em que a trombólise não tem utilidade. A neuroimagem tem sido uma das ferramentas que mais tem proporcionado progressos na precisão da seleção de pacientes, ajudando a predizer quais os pacientes se beneficiarão do tratamento trombolítico.

\section{Registro da oclusão arterial}

O objetivo principal do tratamento trombolítico é a recanalização precoce de uma artéria intracraniana ocluída. $\mathrm{O}$ candidato ideal à trombólise é aquele com um severo déficit neurológico em que se identifica uma oclusão de grande vaso sem sinais precoces de infarto no parênquima cerebral. Apesar de não ser indispensável, a identificação de oclusão arterial é altamente recomendável ${ }^{61}$.

Um dos métodos mais disponíveis para o acesso não invasivo do status neurovascular intra- e extracraniano é o ecodoppler, que permite não só a identificação da oclusão arterial pré-tratamento, mas possibilita também a monitorização do processo de recanalização ${ }^{62}$. 0 grupo de pesquisadores da Universidade de Texas-Houston liderado por Andrei Alexandrov realizou vários estudos demonstrando excelente acurácia do DTC na identificação de oclusão arterial e acompanhamento da recanalização após trombólise, além de ótima correlação com os achados da angiografia digital ${ }^{62-64}$. O DTC tem a vantagem do baixo custo e de ser portátil, podendo ser realizado à beira do leito do paciente quantas vezes for necessário, mas apresenta potenciais desvantagens em relação a outros métodos de neuroimagem: depende muito da experiência do examinador e em até $10 \%$ dos indivíduos a janela de insonação não é adequada ${ }^{65}$.

Da mesma forma que o DTC, a angiografia por TC ou por ressonância magnética (RM) são métodos reconhecidamente sensíveis para a detecção de oclusão intra-arterial nos segmentos proximais dos principais vasos intracranianos, mas são pouco sensíveis na detecção de oclusões em ramos mais distais ${ }^{61,66}$. A angiografia é outra ferramenta de grande valor, mas seu uso deve ser habitualmente reservado aos pacientes candidatos a trombólise intra-arterial após análise não invasiva do status neurovascular ${ }^{61}$.

\section{Avaliação do parênquima cerebral}

O estudo NINDS usou como único critério de neuroimagem para exclusão de pacientes a presença de hemorragia na TC ${ }^{13}$. O estudo ECASS analisou a presença de sinais precoces de infarto na TC e o impacto sobre o prognóstico ${ }^{67-69}$. Os sinais de infarto precoce mais comuns são hipodensidade do parênquima cerebral, desaparecimento da fita insular (borramento da interface entre córtex, substância branca e núcleos da base), apagamento de sulcos por edema, e o sinal hiperdenso da artéria cerebral média ${ }^{70,71}$. O tratamento trombolítico não se mostrou eficaz na presença de hipodensidade precoce maior que $1 / 3$ da extensão do território da artéria cerebral média, e ainda aumentou o risco de hemorragia intracraniana ${ }^{68}$. Já é um consenso entre os especialistas em doenças cerebrovasculares que pacientes com sinais extensos de hipodensidade à TC não devem ser submetidos ao tratamento trombolítico devido ao grande risco de hemorragia intracraniana, mesmo estando com menos de três horas do início dos sintomas. Quanto aos sinais precoces mais sutis de infarto, ainda não se pode definir com segurança se estes têm real impacto no resultado do tratamento trombolítico. Além disso, a detecção destes sinais por neurologistas e radiologistas têm baixa uniformidade e sensibilidade, o que os torna ferramentas pouco acuradas na seleção de pacientes ${ }^{72}$. Recentemente foi criado um novo sistema de pontuação em que o território da artéria cerebral média é dividido em dez regiões para melhor definir a extensão de sinais de infarto precoce (ASPECTS), mas a sua aplicabilidade tem sido questionada ${ }^{73,74}$.

Um dos conceitos mais relevantes no tratamento do AVC na fase hiperaguda é o de penumbra isquêmica. Este tecido potencialmente viável deve ser distinguido do tecido já com morte irreversível (centro do infarto) e do tecido que não está em risco de desenvolver lesão apesar de apresentar uma leve hipoperfusão ${ }^{75}$.

A RM, particularmente as técnicas ponderadas em difusão e perfusão, revolucionaram o papel da neuroimagem na avaliação dos pacientes com doença cerebrovascular aguda ${ }^{76}$. As imagens ponderadas em difusão refletem o status bioenergético do cérebro, enquanto as imagens ponderadas em perfusão refletem o status hemodinâmico. A análise conjunta destas duas técnicas propicia um modelo bastante atraente para a delimitação da área de penumbra isquêmica: a anormalidade de difusão representaria o tecido com lesão irreversível enquanto a margem externa da anormalidade perfusional representaria a periferia da região de penumbra isquêmica. A região de mismatch, que chamaremos neste artigo de discordância difusão/ perfusão (DDP), é aquela com perfusão alterada, mas sem alteração de difusão, e representaria a região de penumbra isquêmica ${ }^{77}$ (figura 1).

Há atualmente um robusto corpo de evidências que apóiam o modelo de DDP para a identificação da penumbra isquêmica. Em pacientes não tratados, anormalidades de difusão precocemente discordantes das de perfusão evoluem rapidamente para as dimensões da anormalidade perfusional inicial ${ }^{78}$. Por 
outro lado, em pacientes em que a reperfusão foi possível, há inibição do crescimento da anormalidade de difusão 79,80. A duração da DDP depende de inúmeros fatores, entre eles talvez o mais importante seja a competência da circulação colateral ${ }^{81}$. Bastante relevante é o fato de ainda se poder observar DDP mesmo após 24 horas do início do processo isquêmico, o que sugere que em pacientes selecionados, a janela temporal para se atuar na preservação da região de penumbra isquêmica seja maior que as tradicionais 3 horas ${ }^{82}$.

O modelo de penumbra isquêmica pela DDP, entretanto, não é exato. Há dados suficientes demonstrando que a extensão da anormalidade de perfusão superestima a região de penumbra isquêmica ao incluir regiões de oligoperfusão benigna ${ }^{83,84}$. Além disso, as anormalidades de difusão precoces são parcialmente reversíveis, sugerindo que as mesmas refletem uma combinação de tecido com infarto irreversível e penumbra isquêmica ${ }^{85-87}$. Vários grupos de pesquisa têm investido no aperfeiçoamento deste modelo através de análises envolvendo múltiplas variáveis obtidas de diferentes aquisições de imagens de RM, no sentido de obter uma delimitação mais exata da penumbra isquêmica ${ }^{88,89}$.

Contudo, o modelo atual ainda é o que se tem de melhor para avaliar um paciente na fase aguda do AVC, pois propicia na maior parte das vezes uma estimativa rápida da penumbra isquêmica e uma maneira prática de selecionar candidatos à terapia trombolítica (figura 2). Caplan e colaboradores chegam a considerar a decisão de trombólise exclusivamente guiada pela TC sem contraste como uma estratégia do tipo "roleta russa" 90. A simples exclusão de hemorragia ou sinais precoces de edema extenso pela TC não é suficiente para prevenir a indicação de trombólise em pacientes com severo déficit neurológico, mas com quadros que sabidamente não respondem bem ao tratamento trombolítico (e.g.; outros diagnósticos clínicos que mimetizam um AVC).

Apesar da menor disponibilidade da RM em comparação à TC, a RM na avaliação do AVC hiperagudo já é uma rotina em vários centros acadêmicos, e é crescente o número de hospitais que vêm disponibilizando serviço de RM em regime 24 horas. Os protocolos de RM já são bastante rápidos, incluindo a angio-RM, e não representam um fator relevante de retardo para o início da trombólise. Além disso, já há evidências de que a RM é capaz de detectar hemorragia intraparenquimatosa hiperaguda de forma acurada, sugerindo que a TC possa vir a ser dispensada para exclusão de hemorragia ${ }^{91,92}$. Talvez o maior impacto do modelo de DDP no AVC seja a possibilidade de otimizar a seleção de candidatos à trombólise, mesmo numa janela temporal maior que 3 horas. Um estudo de meta-análise já demonstrou eficácia da trombólise endovenosa em janela temporal de 3-6 horas ${ }^{25}$ e já há algumas evidências de que a janela temporal pode ser melhor "guiada" pela análise da DDP ${ }^{93}$. Futuros estudos randomizados definirão se a extensão da janela temporal com base na DDP refletirá num melhor prognóstico dos pacientes.

O fato da maioria dos serviços que prestam atendimento ao paciente com AVC na fase aguda não dispor de RM, tem incentivado o desenvolvimento de novas técnicas para aquisição de imagens pela TC, que sejam mais sensíveis na detecção de alterações isquêmicas mais precoces não observadas na TC convencional ${ }^{94-96}$. Estas técnicas utilizam injeção de contraste, possibilitando a distinção entre áreas bem perfundidas das mal perfundidas, com o potencial de oferecer informações comparáveis aos do modelo DDP da RM ${ }^{94}$.

Apesar do PET (tomografia por emissão de positrons) e o SPECT (tomografia computadorizada por emissão de fótons únicos) permitirem valiosas análises da hemodinâmica cerebral, são modalidades de neuroimagem que atualmente têm aplicabilidade limitada no paciente com AVC na fase hiperaguda, além da baixa disponibilidade ${ }^{97,98}$.

\section{CONCLUSÕES}

Os avanços alcançados na última década no diagnóstico e tratamento das doenças cerebrovasculares fez com que o neurologista quando à frente de um paciente com AVCi deixasse a posição de elegantes localizadores de lesão, para uma atitude voltada à terapia, mais invasiva e também mais otimista.

O grande desafio que temos pela frente não é mais o de definir se o tratamento trombolítico é ou não eficaz. $O$ desafio é saber definir exatamente em quais pacientes o tratamento trombolítico é eficaz, em quais pacientes é ineficaz e em quais ele é prejudicial. A neuroimagem certamente contribuirá sobremaneira para o aprimoramento desta seleção. Paralelamente, observaremos o desenvolvimento de novas drogas trombolíticas mais eficazes, dispositivos endovasculares mais seguros, assim como estratégias de neuroproteção mais efetivas.

A tendência de envelhecimento da população mundial

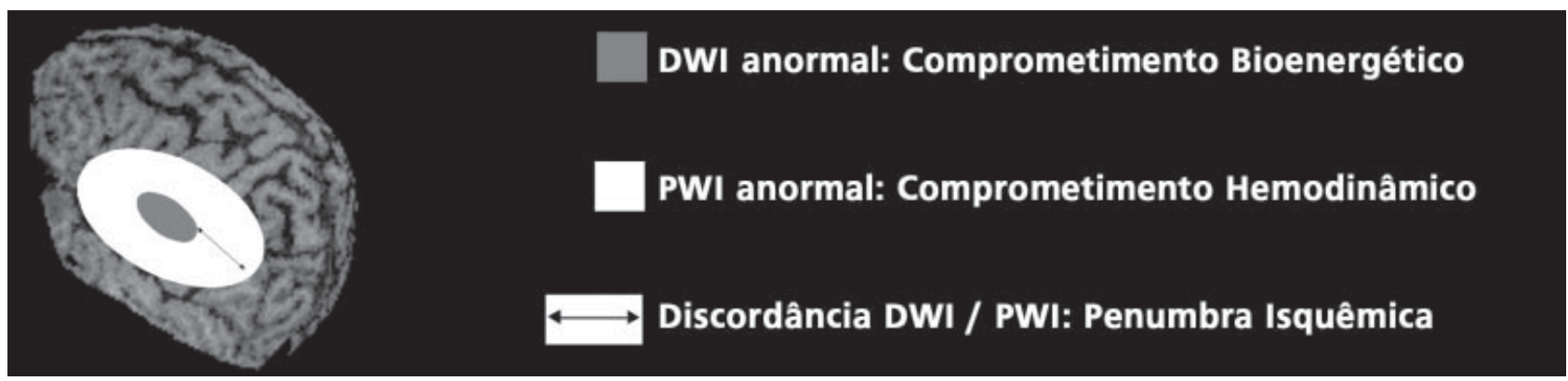

Figura 1: Modelo de discordância nas alterações de difusão e perfusão para penumbra isquêmica. DWI: imagem ponderada em difusão; PWI: imagem ponderada em perfusão. 
fará com que as doenças cerebrovasculares tornem-se um problema de saúde pública ainda maior do que já é no momento. É importante lembrar que o tratamento trombolítico exige a organização de estruturas hospitalares que permitam o fiel cumprimento dos protocolos atualmente aceitos como standard. Devido à curta janela de tempo em que é possível o uso desta terapêutica, várias entidades engajadas no estudo das doenças cerebrovasculares têm-se manifestado no sentido mudar a atitude com relação a estas doenças, tanto por parte da população como também dos profissionais e instituições de saúde envolvidas. A conscientização da população quanto ao reconhecimento de sinais e sintomas de AVC e seu caráter de urgência, bem como a maior disseminação de serviços especializados no atendimento destes doentes permitirá que uma maior parcela da população seja beneficiada pelo tratamento trombolítico, que ainda é sub-utilizado.

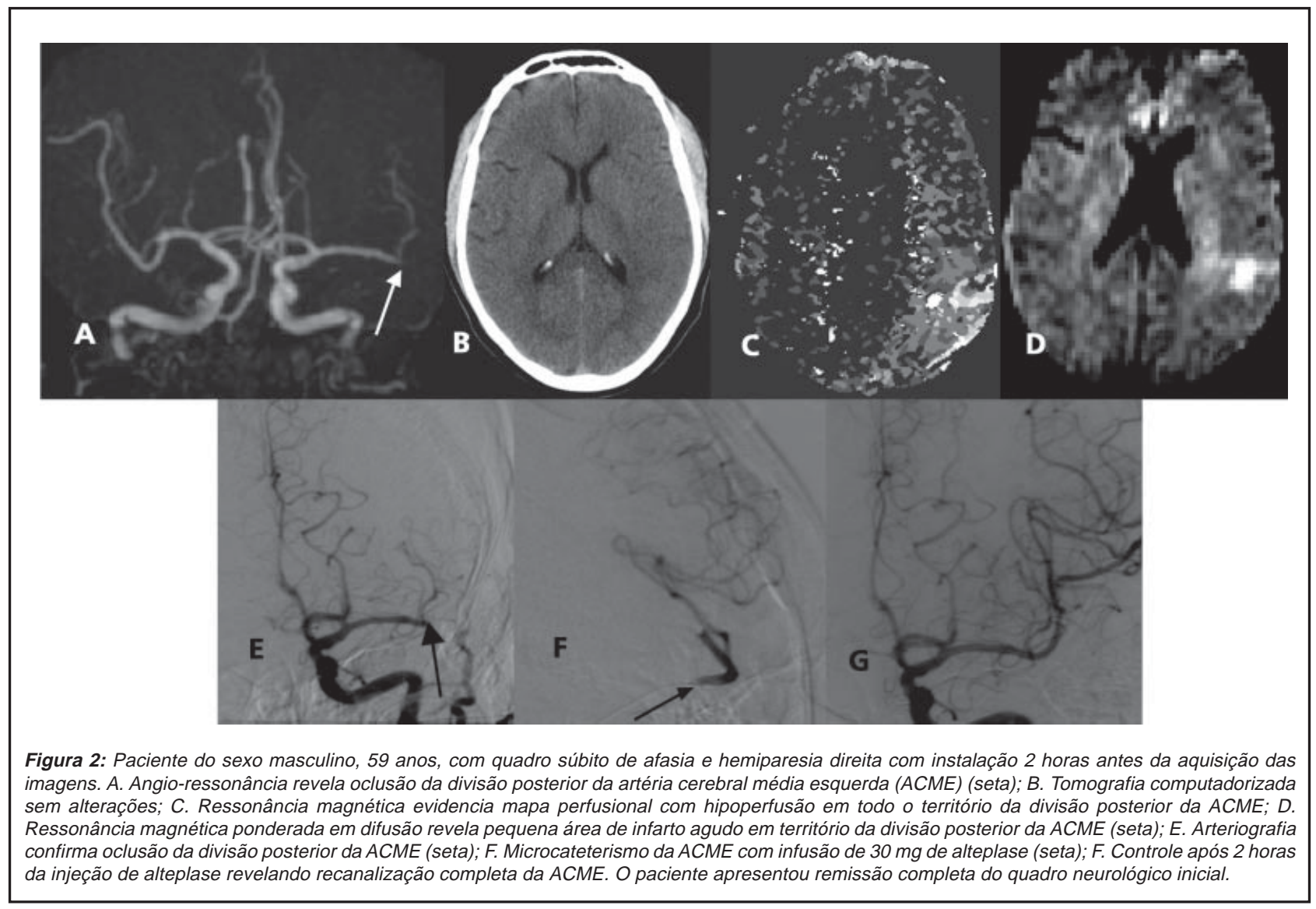

\section{REFERÊNCIAS BIBLIOGRÁFICAS}

1- Sudlow CL, Warlow CP. Comparable studies of the incidence of stroke and its pathological types: results from an international collaboration. International Stroke Incidence Collaboration. Stroke 1997 Mar; 28(3):491-9.

2- Murray CJL, Lopez AD. Mortality by cause for eight regions of the world: Global Burden of Disease Study. Lancet 1997; 349:1269-1276.

3- Sociedade Brasileira de Doenças Cerebrovasculares (SBDCV). Brazilian consensus for the thrombolysis in acute ischemic stroke. Arq Neuropsiquiatr 2002 Sep; 60(3-A):675-80.

4- Caplan LR (ed). Stroke: a clinical approach. Boston: Butterworth-Heinemann, 2000:115-161.

5- Kaluft MA, Saver JL. The acute stroke patient: the first six hours. In Cohen SN (ed), Management of Ischemic Stroke. McGraw-Hill, 2001: 17-52.

6- Zivin JA, Fisher M, DeGirolami U, Hemenway CC, Stashak JA. Tissue plasminogen activator reduces neurological 
damage after cerebral embolism. Science 1985 Dec 13; 230(4731):1289-92.

7- Multicenter Acute Stroke Trial-Italy (MAST-1) Group. Randomised controlled trial of streptokinase, aspirin, and combination of both in treatment of acute ischemic stroke. Lancet 1995; 346:1509-1514.

8- Multicenter Acute Stroke Trial- Europe Study Group. Thrombolytic therapy with streptokinase in acute ischemic stroke. N Engl J Med 1996; 335:145-150.

9- Donnan GA, Davis SM, Chambers BR, et al. Streptokinase for acute ischemic stroke with relationship to time of administration: Australian Streptokinase (ASK) Trial Study Group. JAMA 1996; 276:961-966.

10- Brott TG, Haley Jr EC, Levy DE, Barsan W, Broderick J, Sheppard GL, Spilker hhhhh J, Kongable GL, Massey S, Reed R, Marler JR. Urgent therapy for stroke. Part I. Pilot study of tissue plasminogen activator administered within 90 minutes from onset. Stroke 1992; 23:632-640.

11- Haley Jr EC, Levy DE, Brott TG, Sheppard GL, Wong MCW, Kongable GL, Torner JC, Marler JR. Urgent therapy for stroke. Part II. Pilot study of tissue plasminogen activator administered 91-180 minutes from onset. Stroke 1992; 23:641-645.

12- Brott T, Broderick JP, Kothari R. Thrombolysis for acute ischemic Stroke. In Welch KMA, Caplan LR, Reis DJ, Siesjö BK, Weir B. Primer on cerebrovascular diseases. Academic Press, 1997: 728-731.

13. The National Institute of Neurological Disorders and Stroke rt-PA Stroke Study Group: Tissue plasminogen activator for acute ischemic stroke. N Engl J Med 1995; 333:1581-1587.

14. del Zoppo GJ, Higashida RT, Furlan AJ, et al. PROACT: A phase II randomized trial of recombinant pro-urokinase by direct arterial delivery in acute middle cerebral artery stroke. Stroke 1998; 29:4-11.

15. Hacke W, Kaste M, Fieschi C, et al. Intravenous thrombolysis with recombinant tissue plasminogen activator for acute hemispheric stroke. The European Cooperative Acute Stroke Study. JAMA 1995; 274:1017-1025

16. Hacke W, Kaste M, Fieschi C, et al. Randomized double-blind placebo-controlled trial of thrombolytic therapy with intravenous alteplase in acute ischaemic stroke (ECASS II). Lancet 1998; 352:1245-1251.

17. Clark WM, Wissman S, Albers GW, et al. Recombinant tissue-type plasminogen activator (Alteplase) for ischemic stroke 3 to 5 hous after symptom onset. The ATLANTIS Study: A randomized controlled trial. Alteplase Thrombolysis for Acute Noninterventional Therapy in Ischemic Stroke. JAMA 1999; 282:2019-2026.

18. Suarez JI. Thrombolytic therapy for acute ischemic stroke: current evidence. Syllabi-On-CDROM. American Academy of Neurology 2001.

19. Schellinger PD, Fiebach JB, Mohr A, et al. Thrombolytic therapy for ischemic stroke - A review. Part II - Intra-arterial thrombolysis, vertebrobasilar stroke, phase IV trials, and stroke imaging. Crit Care Med 2001, 29:1819-1825.

20. Becker KJ, Purcell LL, Hacke W, Hanley DF. Vertebrobasilar thrombosis: diagnosis, management, and the use of intra-arterial thrombolytics. Crit Care Med 1996; 24:1729-1742.

21. Furlan A, Higashida R, Wechsler L, et al. Intra-arterial prourokinase for acute ischemic stroke: the PROACT II study: a randomized controlled trial. JAMA 1999; 282:2003-2011.

22. Budzik RF, Pergolizzi RS, Putman CM. Intraarterial thrombolysis for acute ischemic stroke. Seminars in Neurosurgery $2000 ; 11: 107-132$.

23. Grond $\mathrm{M}$, Rudolf $\mathrm{J}$, Schmulling $\mathrm{S}$, et al. Early intravenous thrombolysis with recombinant tissue-type plasminogen activator in vertebrobasilar ischemic stroke. Arch Neurol 1998; 55:466-469.

24. Hacke W, Brott T, Caplan L, et al. Thrombolysis in acute ischemic stroke: Contolled trials and clinical experience. Neurology 1999; 53:S3-14.

25. Wardlaw JM, del Zoppo G, Yamaguchi T. Thrombolysis for acute ischaemic stroke. In: The Cochrane Library, Issue 4. Oxford: Update Software; 2001.

26. Qureshi Al, Suri MFK. New agents for thrombolysis: the third generation

thrombolytics. Syllabi-ON-CDROM. American Academy of Neurology, 2001.

27. Sherman DG, Atkinson RP, Chippendale T, et al. Intravenous ancrod for treatment of acute ischemic stroke: the STAT study: a randomized controlled trial. Stroke Treatment with Ancrod Trial. JAMA. 2000 May 10; 283(18):2395-403.

28. Liu M, Counsell C, Zhao XL, Wardlaw J. Fibrinogen depleting agents for acute ischaemic stroke. Cochrane Database Syst Rev. 2003;(3): CD000091.

29. Donnan GA, Howells DW, Markus R, Toni D, Davis SM. Can the time window for administration of thrombolytics in stroke be increased? CNS Drugs 2003; 17(14):995-1011.

30. Thomas GR, Thibodeaux H, Errett CJ, et al. A long-half-life and fibrin-specific form of tissue plasminogen activator in rabbit models of embolic stroke and peripheral bleeding. Stroke 1994 Oct; 25: 2072-8; discussion 2078-9.

31. Zhang RL, Zhang L, Jiang Q, Zhang ZG, Goussev A, Chopp M. Postischemic intracarotid treatment with TNK-tPA reduces infarct volume and improves neurological deficits in embolic stroke in the unanesthetized rat. Brain Res 2000; 878:64-71. 
32. Qureshi AI, Ringer AJ, Suri MFK, Guterman LR, Hopkins LN. Acute intervention for ischemic stroke: present status and future directions. J Endovasc Ther 2000; 7:423-428

33. Smalling RW, Bode C, Kalbfleisch J, et al. More rapid, complete, and stable coronary thrombolysis with bolus administration of reteplase compared with alteplase infusion in acute myocardial infarction. Circulation 1995; 91:2725-2732.

34. Antman EM, Giugliano RP, Gibson CM, et al., for the TIMI 14 Investigators. Abciximab facilitates the rate and extent of thrombolysis. Results of the Thrombolysis in Myocardial In-farction (TIMI) 14 Trial. Circulation 1999; 99:2729-2732.

35. Collet JP, Montalescot G, Lesty C, et al. Effects of abciximab on the architecture of platelet-rich clots in patients with acute myocardial infarction undergoing primary coronary intervention. Circulation 2001; 103:2328-2331.

36. The abciximab in ischemic stroke investigators. Abciximab in acute ischemic stroke: A randomized, double-blind, placebo-controlled, dose-escalation study. Stroke. 2000; 31:601-609.

37. Houdart E, Woimant F, Chapot R, Mounayer C, Soria C, Merland J. Thrombolysis of extracranial and intracranial arteries after IV abciximab. Neurology 2001; 56:1582-84.

38. Lee KY, Heo JH, Lee SI, Yoon PH. Rescue treatment with abciximab in acute ischemic stroke. Neurology 2001; 56:1585-1587.

39. Heo JH, Lee KY, Kim SH, Kim DI. Immediate reocclusion following a successful thrombolysis in acute stroke: A pilot study. Neurology 2003; 60:1684-1687.

40. Lewandowski C, Frankel M, Tomsick T, et al. Combined intravenous and intra-arterial r-tPA versus intraarterial therapy of acute ischemic stroke: Emergency Management of Stroke (EMS) Bridging Trial. Stroke 1999; 30:2598-2605 41. Ernst R, Pancioli A, Tomsick $\mathrm{T}$, et al. Combined intravenous and intra-arterial recombinant tissue plasminogen activator in acute ischemic stroke. Stroke 2000; 31:2552-2557.

42. Keris V, Rudnicka S, Vorona V, Enina G, Tilgale B, Fricbergs J. Combined intraarterial/intravenous thrombolysis for acute ischemic stroke. AJNR 2001; 22:352-358.

43. Response to intra-arterial and combined intravenous and intra-arterial thrombolytic therapy in patients with distal internal carotid artery occlusion. Stroke 2002; 33:1821-1827.

44. Kimura M, lijima S. Kobayashi K, Furuhata H. Evaluation of the thrombolytic effect of tissue-type plasminogen activator with ultrasound irradiation: in vitro experiment involving assay of the fibrin degradation products from the clot. Biol Pharm Bull 1994; 17: $126-130$.

45. Lauer CG, Burge R, Tang DB, Bass BG, Gomez ER, Alving BM. Effect of ultrasound on tissue-type plasminogen activator-induced thrombolysis. Circulation 1992; 86:1257-1264.

46. Suchkova V, Siddiqi FN, Carstensen EL, Dalecki D, Child S, Francis CW. Enhancement of fibrinolysis with 40-kHz ultrasound. Circulation 1998; 98:1030 -1035.

47. Alexandrov AV. Ultrasound-enhanced thrombolysis for stroke: clinical significance. European Journal of Ultrasound 2002; 16:131-140.

48. Alexandrov AV, Demchuk AM, Felberg RA, et al. High rate of complete

recanalization and dramatic clinical recovery during tPA infusion when continuously monitored with $2 \mathrm{MHz}$ transcranial Doppler monitoring. Stroke 2000; 31:610 -614.

49. Blacker D. High rate of complete recanalization and dramatic clinical recovery during tPA infusion when continuously monitored with 2-MHz transcranial Doppler monitoring. Stroke 2000 Dec; 31(12):3079-3083

50. Behrens S, Spengos K, Daffertshofer M, Schroeck H, Dempfle CE, Hennerici M. Transcranial ultrasound-improved thrombolysis: diagnostic vs. therapeutic ultrasound. Ultrasound Med Biol 2001; 27:1683-1689.

51. Cintas P, Le Traon AP, Larrue V. High rate of recanalization of middle cerebral artery occlusion during 2-MHz transcranial color-coded Doppler continuous monitoring without thrombolytic drug. Stroke 2002; 33: 626-628.

52. Qureshi AI, Siddiqui AM, Suri MF, et al. Aggressive mechanical clot disruption and low dose intraarterial third generation thrombolytic therapy for ischemic stroke: a prospective study. Neurosurgery 2002;51:1319 -1327.

53. Wikholm G. Mechanical Intracranial Embolectomy. A Report of Two Cases.

Interventional Neuroradiology 1998: 4:159-164.

54. Lutsep HL, Clark WM, Nesbit GM, Kuether TA, Barnwell SL. Intraarterial suction thrombectomy in acute stroke. AJNR 2002; 23:783-786.

55. Nakano S, Yokogami K, Ohta H, Goya T, Wakisaka S. Direct percutaneous transluminal angioplasty for acute embolic middle cerebral artery occlusion: report of two cases. Int J Angiol 1997; 6: 254-256.

56. Nakano S, Yokogami K, Ohta H, Yano T, Ohnishi T. Direct percutaneous transluminal angioplasty for acute middle cerebral artery occlusion. AJNR 1998; 19: 767-772.

57. Tsai FY, Berberian B, Matovich V, Lavin M, Alfieri K. Percutaneous transluminal angioplasty adjunct to thrombolysis for acute middle cerebral artery rethrombosis. AJNR 1994; 15: 1823-1829. 
58. Nakano S, Iseda T, Yoneyama T, Kawano H, Wakisaka S. Direct Percutaneous Transluminal Angioplasty for Acute Middle Cerebral Artery Trunk Occlusion An Alternative Option to Intra-arterial Thrombolysis. Stroke 2002 Dec; 33:2872-6.

59. Gupta R, Schumacher HC, Mangla S, et al. Urgent endovascular revascularization for symptomatic intracranial atherosclerotic stenosis. Neurology. 2003 Dec 23; 61(12): 1729-35.

60. Ringer AJ, Qureshi AI, Fessler RD, Guterman LR, Hopkins LN. Angioplasty of intracranial occlusion resistant to thrombolysis in acute ischemic stroke. Neurosurgery 2001; 48:1282-8; discussion 1288-90.

61. Schellinger PD, Fiebach JB, Hacke W. Imaging-based decision making in thrombolytic therapy for ischemic stroke. Present status. Stroke 2003; 34:575-583.

62. Burgin WS, Malkoff M, Felberg RA, et al. Transcranial doppler ultrasound criteria for recanalization after thrombolysis for middle cerebral artery stroke. Stroke 2000; 31:1128-1132.

63. Demchuk AM, Christou I, Wein TH, et al. Specific transcranial doppler flow findings related to the presence and site of arterial occlusion. Stroke 2000; 31:140-146.

64. Christou I, Alexandrov AV, Burgin WS, et al. Timing of recanalization after tissue plasminogen activator therapy determined by transcranial doppler correlates with clinical recovery from ischemic stroke. Stroke 2000; 31:1812-1816.

65. Markus HS. Transcranial doppler ultrasound. J Neurol Neurosurg Psychiatry 1999; 67:135-137.

66. Knauth M, Kummer Rv, Jansen O, Haehnel S, Doerfler A, Sartor K. Potential of CT angiography in acute ischemic stroke. AJNR Am J Neuroradiol 1997; 18:1001-1010.

67. von Kummer R, Nolte PN, Schnittger H, Thron A, Ringelstein EB. Detectability of cerebral hemisphere ischaemic infarcts by CT within 6h of stroke. Neuroradiology 1996; 38:31-33.

68. von Kummer R, Meyding-Lamade U, Forsting M, et al. Sensitivity and prognostic value of early CT in occlusion of the middle cerebral artey trunk. AJNR 1994; 15:9-15.

69. von Kummer R, Allen KL, Holle R, et al. Acute stroke: usefulness of early CT findings before thrombolytic therapy. Radiology 1997; 205:327-333.

70. Grond M, von Kummer R, Sobesky J, et al. Early computer tomography abnormalities in acute stroke. Lancet 1997; 350:1595-1596.

71. Gacs G, Fox AJ, Barnett HJM, Vinuela F. CT visualisation of intracranial arterial thromboembolism. Stroke 1982; 14:756-762.

72. Schriger DL, Kalafut M, Starkman S, Krueger M, Saver JL. Cranial computed tomography interpretation in acute stroke: physician accuracy in determining eligibility for thrombolytic therapy. JAMA 1998; 279:1293-1297.

73. Barber PA, Demchuk AM, Zhang J, Buchan AM. Validity and reliability of a quantitative computed tomography score in predicting outcome of hyperacute stroke before thrombolytic therapy. Lancet 2000; 355:1670-1674.

74. Nawashiro H, Fukui S. Intra-arterial thrombolysis for hyperacute stroke. Lancet 2000; 356:1111-1112.

75. Hakim AM. The cerebral ischemic penumbra. Can J Neurol Sci 1987; 14:557-559.

76. Baird AE, Warach S. Magnetic resonance imaging of acute stroke. J Cereb Blood Flow Metab 1998; 18:583-609.

77. Kidwell CS, Alger JR, Saver JL. Beyond mismatch evolving paradigms in imaging the ischemic penumbra with multimodal magnetic resonance imaging. Stroke 2003; 34:2729-2735.

78. Baird AE, Benfield A, Schlaug G, et al. Enlargement of human cerebral ischemic lesion volumes measured by diffusion-weighted magnetic resonance imaging. Ann Neurol 1997; 41:581-589.

79. Jansen $\mathrm{O}$, Schellinger $\mathrm{P}$, Fiebach $\mathrm{J}$, et al. Early recanalisation in acute ischaemic stroke saves tissue at risk defined by MRI. Lancet 1999; 353:2036-2037.

80. Parsons MW, Barber PA, Chalk J, et al. Diffusion- and perfusion- weighted MRI response to thrombolysis in stroke. Ann Neurol 2002; 51:28-37.

81. Marchal G, Beaudouin V, Rioux P, et al. Prolonged persistence of substantial volumes of potentially viable brain tissue after stroke: a correlative PET-CT study with voxel-based data analysis. Stoke 1996; 27:599-606.

82. Darby DG, Barber Pa, Gerraty RP, et al. Pathophysiological topography of acute ischemia by combined diffusionweighted and perfusion MRI. Stroke 1999; 30:2043-2052.

83. Rohl L, Ostergaard L, Simonsen CZ, et al. Viability thresholds of ischemic penumbra of hyperacute stroke defined by perfusion-weighted MRI and apparent diffusion coefficient. Stroke 2001; 32: 1140-1146.

84. Parsons MW, Yang Q, Barber PA, et al. Perfusion magnetic resonance imaging maps in hyperacute stroke: relative cerebral blood flow most accurately identifies tissue destined to infarct. Stroke 2001; 32:1581-1587.

85. Kidwell CS, Saver JL, Mattiello J, et al. Thrombolytic reversal of acute human cerebral ischemic injury shown by diffusion/perfusion magnetic resonance imaging. Ann Neurol 2000; 47:462-469.

86. Chalela JA, Ezzeddine MA, Callabrese TM, et al. Diffusion and perfusion changes two hours after intravenous rt-PA therapy: a preliminary report. Stroke 2002; 33:356-357. 
87. Kidwell CS, Saver JL, Starkman S, et al. Late secondary ischemic injury in patients receiving intraarterial thrombolysis. Ann Neurol 2002; 52:698-703.

88. Wu O, Koroshetz WJ, Ostergaard L, et al. Predicting tissue outcome in acute human cerebral ischemia using combined diffusion- and perfusion-weighted MR imaging. Stroke 2001; 32: 933-942.

89. Jacobs MA, Mitsias $P$, Soltanian-Zadeh $\mathrm{H}$, et al. Multiparametric MRI tissue characterization in clinical stroke with correlation to clinical outcome: part 2. Stroke 2001; 32:950-957.

90. Caplan LR, Mohr JP, Kistler JP, Koroshetz W. Should thrombolytic therapy be the first-line treatment for acute ischemic stroke? Thrombolysis- not a panacea for ischemic stroke. N Engl J Med. 1997;337:1309-10

91. Schellinger PD, Jansen O, Fiebach JB, Hacke W, Sartor K. A standardized MRI stroke protocol: comparison with CT in hyperacute intracerebral hemorrhage. Stroke 1999; 30:765-768.

92. Fiebach JB, Schellinger PD, Gass A, et al. Stroke magnetic resonance imaging is accurate in hyperacute intracerebral hemorrhage: A multicenter study on the validity of stroke imaging. Stroke 2004 35: 502 - 506

93. Röther J, Schellinger PD, Gass A, et al. Effect of intravenous thrombolysis on MRI parameters and functional outcome in acute stroke $<6$ hours. Stroke 2002; 33:2438-2445.

94. Schramm P, Schellinger PD, Fiebach JB, et al. Comparison of CT and CT angiography source images with diffusion-weighted imaging in patients with acute stroke within 6 hours after onset. Stroke 2002; 33:2426-2432.

95. Lev MH, Segal AZ, Jeffery Farkas J, et al. Utility of perfusion-weighted CT imaging in acute middle cerebral artery stroke treated with intra-arterial thrombolysis. Prediction of final infarct volume and clinical outcome. Stroke 2001; 32:2021-2028.

96. Koenig M, Kraus M, Theek C, Klotz E, Gehlen W, Heuser L. Quantitative assessment of the ischemic brain by means of perfusion-related parameters derived from perfusion CT. Stroke 2001; 32:431-437.

97. Heiss WD, Grond M, Thiel A, et al. Tissue at risk of infarction rescued by early reperfusion: a positron emission tomography study in systemic recombinant tissue plasminogen activator thrombolysis of acute stroke. J Cereb Blood Flow Metab 1998; 18:1298-1307.

98. Berrouschot J, Barthel H, Hesse S, Koster J, Knapp WH, Schneider D. Differentiation between transient ischemic attack and ischemic stroke within the first six hours after onset of symptoms by using 99 mTc-ECD-SPECT. J Cereb Blood Flow Metab 1998;18:921-929. 